\title{
UWB Cooperatif Radar for Localization and Communication Dedicated to Guided Transport
}

\author{
T. Tahri \\ Institue of Electronics Microeletectronics and Nanotechnology, Iemn/Doae, Valenciennes, France \\ Tarik.Tahri@univ-valenciennes.fr/tatahri@gmail.com \\ Y. Elhillali, L. Sakkila, A. Rivenq \\ Institue of Electronics Microeletectronics and Nanotechnology, Iemn/Doae, Valenciennes, France \\ Yassin.elhillali@univ-valenciennes.fr, laila.sakkila@univ-valenciennes.fr, atika.rivenq@univ-valenciennes.fr
}

\begin{abstract}
Wireless technology for communication and localization in train applications are widely used. Ultra wide band appears as a very suitable technology for this kind of application, due to its large bandwidth, also to its good resistance to the interference and to multipath. In this paper, a new system dedicated to railway transport, based on UWB technology is presented. The originality of this study is combination of the two main functionalities, localization and communication providing a high data rate. The sensor, in order to detect the position of vehicles, uses a matched digital correlation receiver. To allow a multi user access and to combine the two functionalities, two original multiplexing techniques called SSS2 (Sequential Spreading Spectrum technique) and CPM (Code Position Modulation) are performed, in addition to other parameters like used waveform and orthogonal codes.
\end{abstract}

Index Terms - Radar, Localization, Communication, Multiplexing, UWB, Multiple Access

\section{INTRODUCTION}

For a long time, a radio transmission between infrastructure and trains has been non-existing, so the only information which the driver on board his vehicle has at his disposal was the ones provided by signaling. Today, vast changes are afoot in the field of telecommunications between fixed equip ment and trains, with the development of GSM radio networks and satellite-aided localization of vehicles [1], among others. Control, command and communication trains applications are the major areas for railway operators. But presently, no one of existing systems for rail applications ensures localization and data communication with a high data rate.

This paper, presents the implementation of an original sensor using Ultra Wide band technology, allowing the both functionalities in the same system. It aims to improve reliability, safety and security on rails. The system is based on the principle of cooperative radar using ultra wide band technology and using a numerical correlation receiver. To separate localization signal from communication one, different techniques has been developed.

The advantages of this system are that it not only provides not only a real time distance between train and target, but also allows high data rate transmis sion between sensors, which could be helpful to develop many applications among which exchange of informat ion such as video records, in order to increase safety feeling and quality of service inside train.

The first part of this paper, the proposed co-operative radar is presented. The used codes for localization are discussed in the second part. After that, the proposed techniques of multiplexing are introduced in the third part. Finally a performance comparison is performed in the last part.

\section{PROPOSED SYSTEM}

The rail is in perpetual evolution, his research axes are mainly motivated by a growing need in term of data rates, quality of transmission, accuracy and reliability of the localization. However, it remains slowed by a frequentiel environment, which is more and more polluted (crowded spectrum).

In particular, Ultra Wide Band technology with its nature and its bandwidth represents a good resistance to the noise as well as to the multipath, what makes it very adapted to railroad applications. So, thanks to its low spectral density, it is able to coexist with the already existing systems.

In this context, the use of the UWB technology to resolve problems related to railways seems a very promising alternative [2]. The proposed system is UWB co-operative radar which provides localization functionalities and data communication features.

The principle of this system is to send a frame, composed of two sub-frames: the first one contains a code $\mathrm{C} 1$ dedicated to the localization, and the second one contains the data bit coded with another code $\mathrm{C} 2$. In order to ensure a regular renewal of the distance measurement, the localization burst is sent periodically. In this paper two coding techniques are presented: the simple spreading spectrum with 2 codes SSS2 and the code position modulation CPM (inspired from PPM modulation [3]), and will allow a multiuser access.

For this purpose, each user has its own associated code. The coding is done by multiplying each code bit by the UWB pulse as done in CDMA technique (Code Division Multiple Access [4]). 


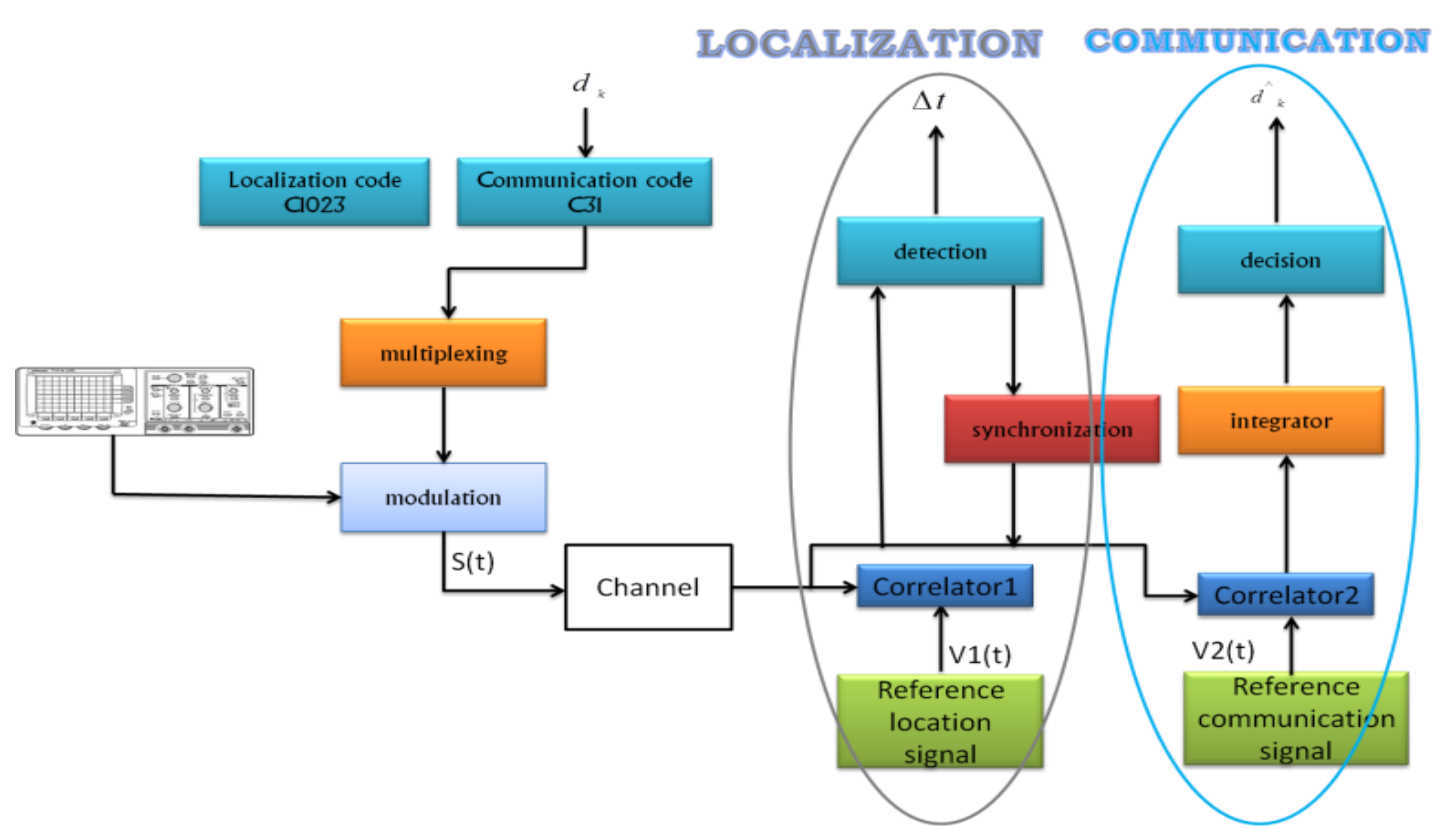

Fig. 1. the proposed bi-functionalities radar based on UWB technology

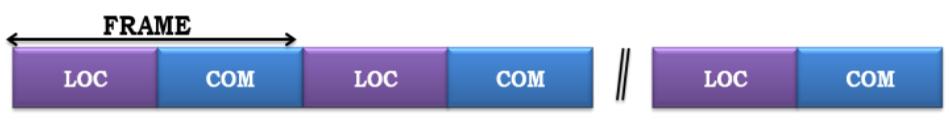

Fig. 2. general structure of transmitted signal

Another way to perform multiple accesses could be done to use waveforms based on orthogonal polynomials. The best-known UWB orthogonal waveforms are Gegenbauer and Hermite polynomials. Therefore, each user has its own associated function order. These orthogonal waveforms associated to codes ensure a double orthogonality [5].

In our system, we use the first multiuser technique (CDMA) with a Gaussian waveform.

To calculate a distance between train and the target,a correlation receiver is used[6]. The correlation result is transferred to the processing unit, which will compare its maximum with a threshold to decide the presence or not of a target in front of the radar [4]. If the target is detected, so the distance is estimated using the following equation:

$$
d=\frac{c . \Delta t}{2}
$$

Where c represents the celerity and $\Delta t$ the delay time.
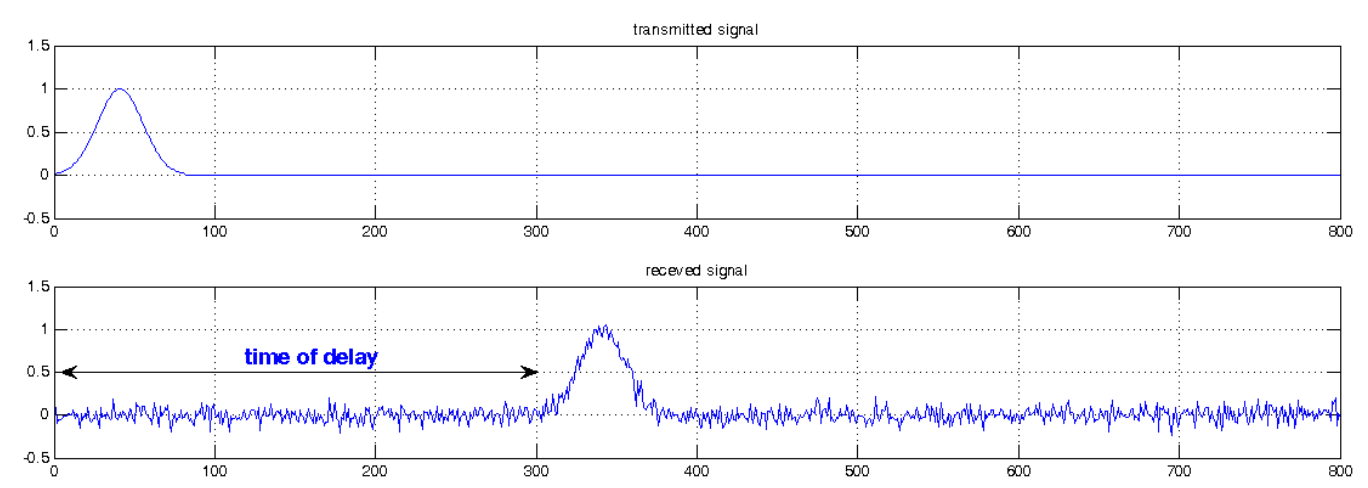

Fig. 4. delay time between the emitted and received pulse

The performance of this localization system is depends onthe choice of code, based on the criteria of orthogonality, ease of generation and the number of codes that compose the family of code, which will be developped in the next part.

\section{CODES AND WAVE FORMS CHOICE}

\section{A. waveforms choice}

In the following paragraphs, different orders of Hermite and Gegenbauer polynomials [7][8][13], which are 
defined respectively by equations 2 and 3 and represented respectively in figures 5 and 6 :

$$
\left.\begin{array}{l}
h_{\mathrm{O}}(t)=e^{-t^{2} / 4} \\
h_{1}(t)=t e^{-t^{2} / 4} \\
h_{2}(t)=\left(t^{2}-1\right) e^{-t^{2} / 4} \\
h_{3}(t)=\left(t^{3}-3 t\right) e^{-t^{2} / 4}
\end{array}\right\}
$$

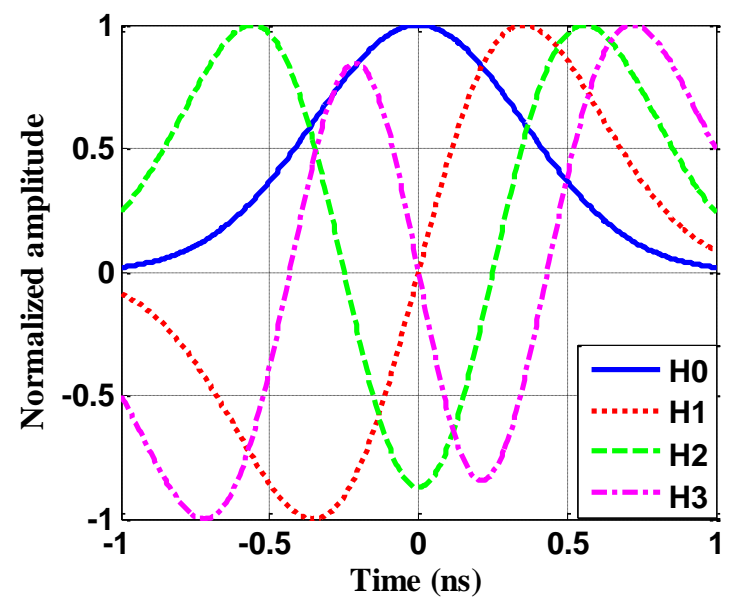

Fig. 5. four first order of Hermite function

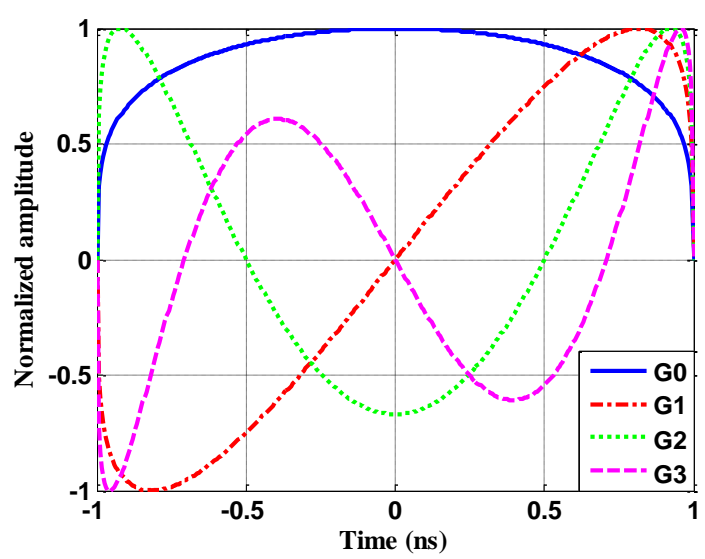

Fig. 6. four first order of Gegenbauer function

After an initial comparison between hermite and Gegenbauer polynomials, the orders that give the best performance for the radar detection functionality according to the best ratio between the dynamics and peak width are selected[4].

In the second stage, the autocorrelation function results, obtained using the selected orders previously and other waveforms, using the Gaussian and monocycle pulses (first derivative of Gaussian function) is compared.

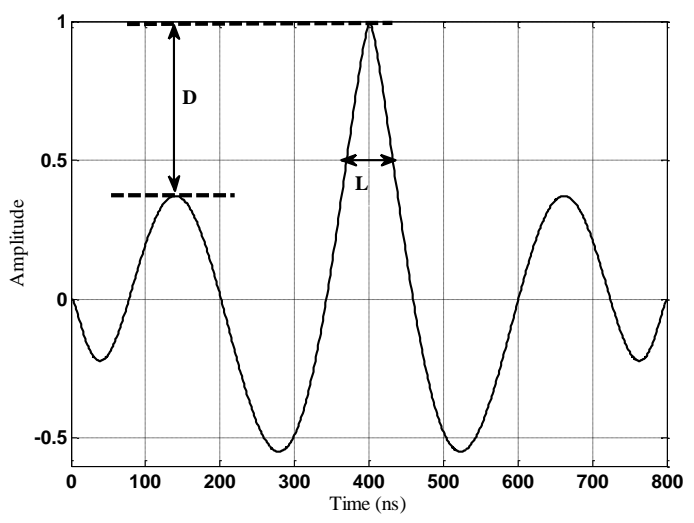

Fig. 7. dynamic definition

The autocorrelation functions of the first four Gegenbauer functions ( $\mathrm{G} 0$ to $\mathrm{G} 3$, with $\beta=1$ ), first four Hermite functions (h0 to h3), Gaussian pulse and monocycle pulse have been computed and the results are summarized in table 1.

The dynamics, peak width and the efficiency which is defined by the following equation [9]:

$$
\text { Efficiency }=\frac{\text { Dynamic }}{\text { peak_width }}
$$

have been recapitulated therefore. The results are given in table 1.

Table 1. efficiencies for the studied waveform

\begin{tabular}{|c|c|c|c|}
\hline Waveform & Dynamics & Peak width & Efficiency \\
\hline $\mathbf{G}_{\mathbf{0}}$ & 1 & 386 & $26.10^{-4}$ \\
\hline $\mathbf{G}_{\mathbf{1}}$ & 1 & 150 & $67.10^{-4}$ \\
\hline $\mathbf{G}_{\mathbf{2}}$ & 0.70 & 89 & $79.10^{-4}$ \\
\hline $\mathbf{G}_{\mathbf{3}}$ & 0.63 & 62 & $102.10^{-4}$ \\
\hline $\mathbf{H}_{\mathbf{0}}$ & 1 & 234 & $43.10^{-4}$ \\
\hline $\mathbf{H}_{\mathbf{1}}$ & 1 & 124 & $81.10^{-4}$ \\
\hline $\mathbf{H}_{\mathbf{2}}$ & 0.61 & 94 & $65.10^{-4}$ \\
\hline $\mathbf{H}_{\mathbf{3}}$ & 0.58 & 74 & $78.10^{-4}$ \\
\hline Gaussian & 1 & 142 & $70.10^{-4}$ \\
\hline Monocycle & 1 & 115 & $87.10^{-4}$ \\
\hline
\end{tabular}

Considering the relationship between the dynamic and width of the correlation peak, these comparisons show that the order 3 of Gegenbauer functions has that the highest efficiency among Gegenbauer functions belongs to the order 3 (G3). A mong Hermite functions, the order 1 (h1) looks the best.

Finally, as seen in table 1, from efficiency point of view, the third order Gegenbauer function seems to be the best, followed by the monocycle pulse, the first order of Hermite function and the Gaussian pulse.

\section{B. codes choice}

The goal is to compare the performance of two codes, Binary Pseudo-Random Sequences (BPRS) and Gold 
codes. To choose the code that gives the best performance on the one hand, and different lengths for each code on the second hand.

This comparis on is based on the results of correlation calculation.

For that, a chain of transmission to send [ $\left[\begin{array}{lll}1 & 1 & -1\end{array}\right]$ is realized, using an AWGN channel. The principle is to encode data with the studied codes (GOLD and BPSR), in the reception, the correlation of the received signal and the codes is calculated.

Figure 8 shows a result of the correlation Gold codes and BPSR code of length 31 .

Moreover, the binary pseudo-random sequences used, combined with the correlation receiver, are very adapted to the detection of signal over noisy communication channel[10].

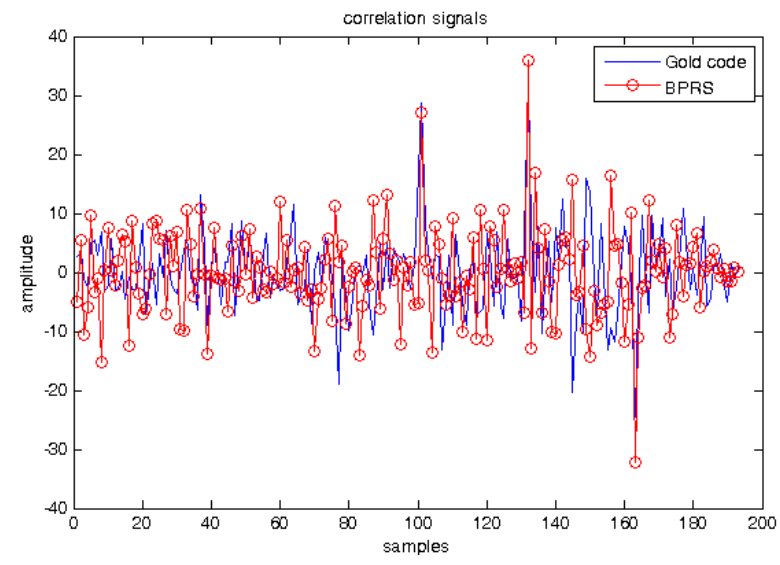

Fig. 8. comparison between Gold code and BPSR correlation

The number of code per family depends on the register length used [7] (Table2).

Table 2. properties of Gold and BPSR codes

\begin{tabular}{ccccc}
\hline family & Code length $\mathrm{N}$ & Code number & Max cross correlation & Max autocorrelation \\
\hline GOLD & $2^{n}-1$ & $2^{n}+1$ & $\left(2^{(n+2) / 2}+1\right) / N$ & 1 \\
BPSR & $2^{n}-1$ & depends & $\left(2^{n / 2}+1\right) / N$ & 1 \\
\hline
\end{tabular}

Table 3. number of orthogonal BPSR codes according to register length

\begin{tabular}{ccccccccc}
\hline Register length & $\mathbf{3}$ & $\mathbf{4}$ & $\mathbf{5}$ & $\mathbf{6}$ & $\mathbf{7}$ & $\mathbf{8}$ & $\mathbf{9}$ & $\mathbf{1 0}$ \\
\hline Code number & 2 & 2 & 6 & 6 & 18 & 16 & 48 & 60 \\
\hline
\end{tabular}

Indeed, the length of the first code 1023 is chosen due to the important number of required codes (60) and in order to increase the range of the system.

The length of the second code affects the communication data rate. In fact, if the choice is a shorter one, the rate will be higher but the robustness will decrease significantly.

Multiple simulations have been done and the length of 31 bits seems to be a good compromise between data rate and quality of transmission.

\section{MultiPleXING TeCHNIQUES}

This part focuses on solutions proposed to develop an original high data rate communication feature comb ined to current localization functionality.

In the interest to perform this combination, two different coding techniques, allowing a continuous refreshing of the localization and ensuring a high data rate for transmission with a suitable BER ( Binary Error Rate) were tested. The proposed multiplexing method is inspired from the DS-CDMA [11] and the PPM (pulse position modulation), it uses families of orthogonal codes, previously studied.

The two techniques differ in the method of coding the data inside the communication sub-frame. These techniques will be detailed and compared in the following paragraphs.

\section{A. The UWB-SSS2 technique}

The first coding technique, untitled SSS2 for Sequential Spectrum Spreading using 2 codes, is inspired from the CDMA technique.

The first spreading with localization code C1023 modulated using antipodal [12] modulation with the appropriate UWB waveform is used to ensure the localization function. The second one is used to code data communication with communication code C31 (with the same modulation) as in classical DS-CDMA. This technique allows to send 33 bits of data between two localization operations.

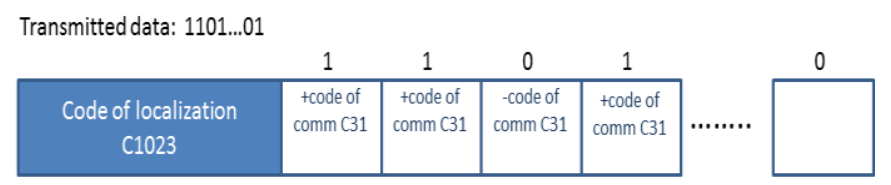

Fig. 9. structure of transmitted signal by the SSS2 method 
The figure 9 shows the structure of the transmitted frame for this technique.

To calculate the distance, the correlation between the received signal and the first reference signal (C1023 modulated) is computed. The correlation peaks allows the synchronization process; then, to recover data, a second correlation between the received signal and the second reference signal (C31 modulated) is performed

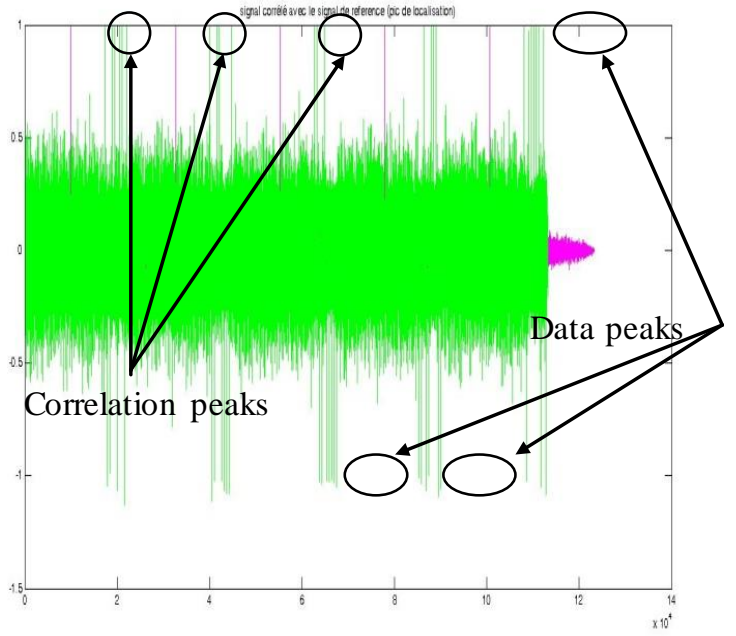

Fig. 10. the two correlation results

\section{B. The UWB-CPM technique}

The second technique is called CPM for Code Position Modulation and is inspired from PPM modulation.
This method consists in coding the data according to the position of the code $\mathrm{C} 31$ inside the communication subframe.

The localization process is the same as proposed in SSS2, except that the data sub-frame will be divided into several sub-frames of length $\mathrm{N}$ (figure 11). In each subframe, several bits ( $\mathrm{N}$ bits) will be coded at the same time using the $\mathrm{C} 31$.

This coding will be done in two steps for each subframe:

$\checkmark$ The MSB (Most Significant Bit) will determinate the polarity of C31 code to be sent.

$\checkmark$ The N-1 LSBs (Lowest Significant Bit), which constitute the remainder of the block, will determinate the position of the polarized code in the sub-frame.

To calculate the distance, the correlation between the received signal and the first reference signal is computed. The correlation peak allows the synchronization to the beginning of the data sub-frames.

In order to detect the presence of data communication frame, a correlation between the rest of the signal and the second reference signal will be calculated for each subframe (we can calculate a circular or linear correlation). The sign of the detected peak gives the MSB (positive for the bit 1 and negative for 0 ). Furthermore, the position of this peak gives the $(\mathrm{N}-1)$ LSBs.

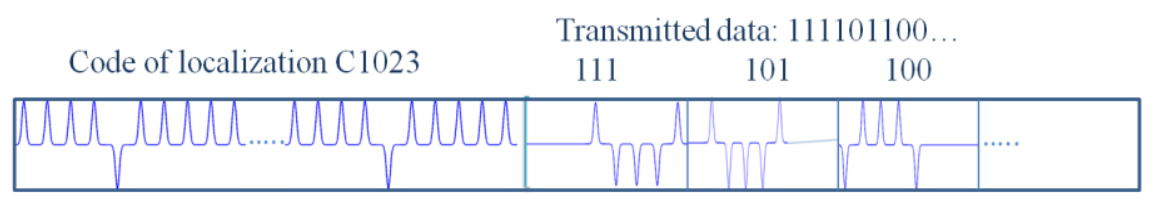

Fig. 11. structure of transmitted signal by the CPM method

However, the circular correlation function of two signals $\mathrm{x}(\mathrm{n})$ and $\mathrm{y}(\mathrm{n})$ is defined by:

$$
r_{x y}=\sum_{k=0}^{N-1} x(k+n) \cdot y(k)
$$

So, its Fast Fourier Transform is:

$$
R_{x y}=X(n) \cdot Y^{*}(n)
$$

Where $X(n)$ and $Y(n)$ is respectively the FFT of $x(n)$ and $y(n)$.

Finally, to obtain $r_{x y}$ we calculate the Inverse Fast Fourier Transform of $R_{x y}$

$$
r_{x y}=\operatorname{IFFT}\left(R_{x y}\right)
$$

The Figure 12 shows the difference between the linear correlation of a SBPR code C31, and the circular correlation of a C31 code and the same C31 code with 10 rotations.

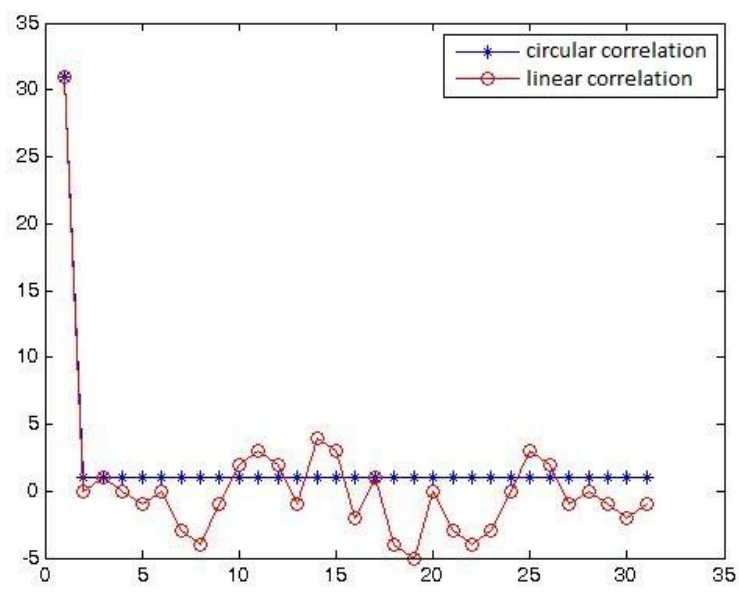

Fig. 12. comparison between linear and circular correlation

Note that the circular corre lation has just two values " 0 " and the length of the correlated signal, what will be an advantage in the reception and make the detection operations easier. 
In addition, the circular corre lation will allow extracting the number of rotation applied in the emission process, with another multiplexing technique called $2 \pi \mathrm{M}$, which will be developed.

\section{Comparison between the two techniques}

As can be seen, the data rate can be affected by the pulse width and the number of bit by block $\mathrm{N}$

$$
\text { Data_rate }_{\max }=\frac{N_{\text {bloc }} * N}{\left(L_{c 1023}+N_{\text {bloc }} * L_{c 31}\right) * T_{\text {pulse }}}
$$

With

$$
N_{b l o c}=\left\lceil\frac{L_{c 1023}}{L_{c 31}+2^{N-1}-1}\right\rceil
$$

Where $L_{c 1023}$ and $L_{c 31}$ are respectively the length of the localization code C1023 and the communication code C31, and $T_{\text {pulse }}$ is the pulse width.

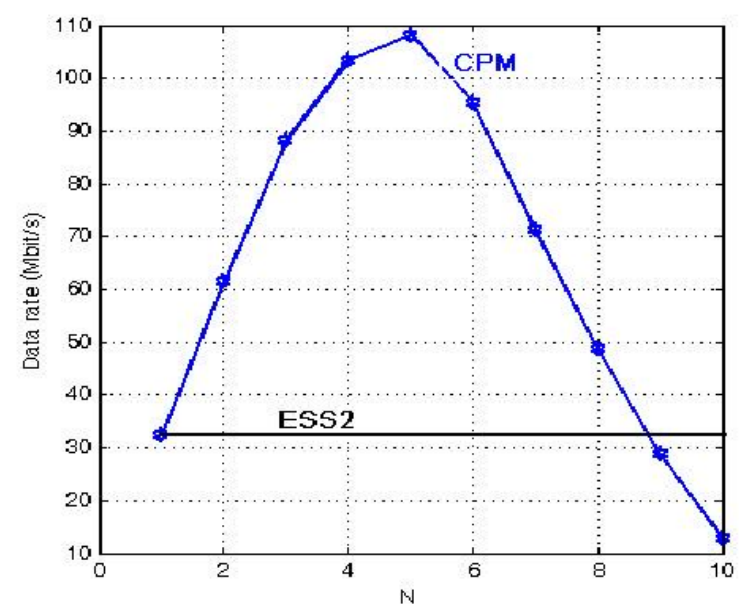

Fig. 13. comparison between ESSS2 and CPM techniques data rate

In the case of CPM method, figure 13 shows that the optimu m of data rate can be obtained with $\mathrm{N}=5$. So, in our simulation the value of $\mathrm{N}$ which optimizes the performance of the systemis used.

Concerning the localization characteristics, both techniques give an identical resolution in distance which is theoretically equal to $0.15 \mathrm{~m}$ depending of the signal frequency used (10GHz).

In order to evaluate the performances of the studied coding techniques in term of data rate and BER, the two methods have been simulated in an AWGN channel.

The figure 14 shows the simulated binary error rate, given for several signal to noise ratio values using the two techniques.

Moreover, a BER of $10^{-4}$ can be reached at SNR equal to $-7 \mathrm{~dB}$ (when the number of coded bit $\mathrm{N}=5$ ) with the CPM technique and $-8.7 \mathrm{~dB}$ with the SSS2.

However, due to the 33 bit data sent, the SSS2 method enables to offer a maximu $\mathrm{m}$ high data rate of about 32.25 Mbits/s (with $T_{\text {pulse }}=0.5 \mathrm{~ns}$ ), while the CPM technique allows a significant flow rate of about $108.10 \mathrm{Mbits} / \mathrm{s}$ with the same value of the pulse width.

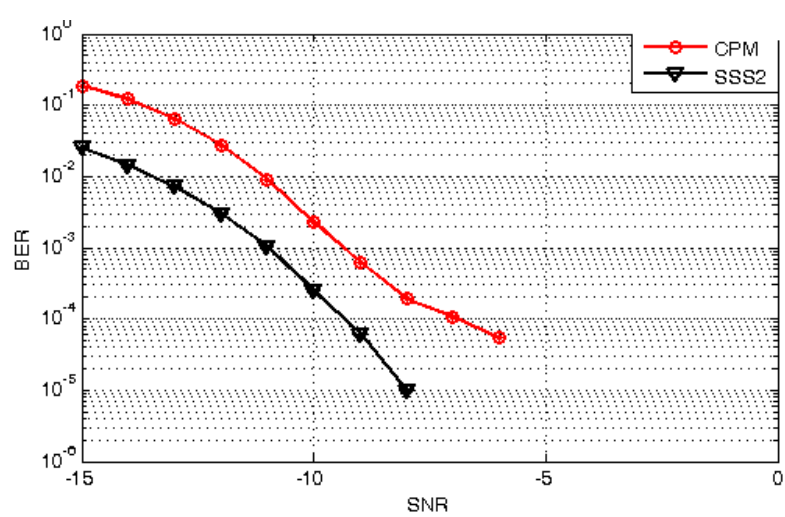

Fig. 14. the BER obtained with SSS2 and CPM techniques

The preliminary simulation results confirm that communication performances of the two coding methods are complementary: the first one, SSS2, offers a good quality of transmission (low values of BER) and $t$

The second one, CPM, offers a high-speed information exchange.

\section{CONCLUSION}

In this paper, cooperative radar based on ultra wide band technology was presented. To ensure localization and communication functions different techniques of multiplexing were presented, such as SSS2 and CPM methods, which are adapted for multi-user needs.

Different parameters were studied, like UWB waveforms and different orthogonal codes. The simulation results show the good performances of this system using BPRS code in term of precision of localization, BER and data rate, which can reach more than $108 \mathrm{Mbits} / \mathrm{s}$.

The study of other techniques to develop this novel and original system is now underway. To improve the robustness to noise, other detection techniques and channelwill also be tested.

\section{REFERENCES}

[1] www.gsmr-conference.com/Press

[2] Elbahhar, F., Rivenq-Menhaj, A. Rouvaen, J.M., and Heddebaut, M. "Inter- Vehicle communication based on Ultra-Wide Band technique," Proc. Tele- com 2001, Casablanca

[3] Tatkeu, C., Elhillali, Y., Rouvean, J.M., 2004. Evaluation of coding methods for development of a radar sensor for localization and communication dedicated to guided transp ort. IEEE VTC'2004, September, Los Angles, USA.

[4] Dixon, R. (Ed), 1976. Spread spectrum techniques. IEEE press, New York.

[5] SAKKILA.L « Study and implementation of an ultra-wideband radar aimed to obstacle detection and recognition in road environments» $\mathrm{PhD}$ thesis, University of Valenciennes and Hainaut Cambrésis, december 2009

[6] Y. E. HILLALI, " study and implementation of communication and localization system, based on spreding spectrum techniques, dedicated to railway transport» $\mathrm{PhD}$ thesis, University of Valenciennes and Hainaut Cambrésis, december 2005 
[7] Lachlan, B.M., Ghavami, M., and Kohno, R., 'Multiple Pulse Generator for Ultra-Wideband Communication using Hermite Polynomial Based Orthogo- nal Pulses,” IEEE Conference on Ultra Wideband Systems and Technology, 2002.

[8] Elbahhar, F., Rivenq-Menhaj, A., Rouvaen, J.M, 'Multiuser Ultra Wide Band communication system based on modified Gegenbauer and Hermite functions," Wireless Personal communications, Volume 34, Issue 3, August 2005

[9] L.SAKKILA."Comparison of classical and orthogonal UWB waveforms for radar applications" $20106^{\text {th }}$ international colloquium on signal processing and its applications, 05/2010

[10] Y.ELHILLALI."Enhanced high data rate communication system using embded cooperative radar for intelligent transp orts systems" Transportation research part C, 2006

[11] S.Pradhan,"A novem orthogonal minimum correlation spreeding code in CDMA systems" I.J.Wirless and Microwave Technologies, 2014, 2, 38-52

[12] M.HAILIANG,'Modeling and performance evaluation of a BPPMUWB system" MSc thesis, Deflft university og technology

[13] ELBAHHAR.F « conception of ultra wide band communication system dedicated to transport» $\mathrm{PhD}$ thesis, University of Valenciennes and Hainaut Cambrésis, november 2003

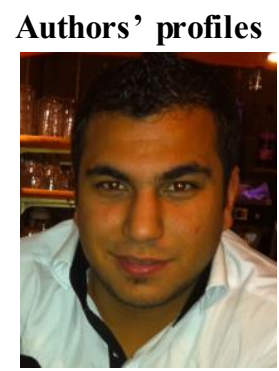

T. TAHRI was born in Morocco in 1987. He received his Master degree from the university of Valenciennes and Hainaut Cambresis, Valenciennes, France in 2010. He is working toward his $\mathrm{PhD}$ degree in electronic with the same university. His primary research interests include the areas of signal processing, cooperative radar systems and communication systems applied to intelligent transportation.

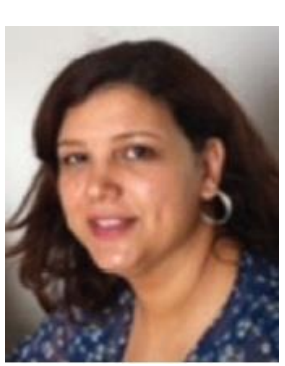

systems
Atika Rivenq-Menhaj was born in 1970. She received her Diploma of Engineering and the M.S. degree in 1993 and then her Ph.D. degree in 1996, from the University of Valenciennes (France). She actually is Professor in electronics at this university. Her primary interest is in signal processing applied to intelligent transportation systems and telecommunication

How to cite this paper: T. Tahri, Y. Elhillali, L. Sakkila, A. Rivenq,"UWB Cooperatif Radar for Localization and Communication Dedicated to Guided Transport", International Journal of Intelligent Systems and Applications(IJISA), vol.6, no.10, pp.27-33, 2014. DOI: 10.5815/ijisa.2014.10.04 